PROCEEDINGS OF THE AMERICAN MATHEMATICAL SOCIETY

Volume 124, Number 6, June 1996

\title{
A RECIPROCITY LAW FOR CERTAIN FROBENIUS EXTENSIONS
}

\author{
YUANLI ZHANG \\ (Communicated by Dennis A. Hejhal)
}

\begin{abstract}
Let $E / F$ be a finite Galois extension of algebraic number fields with Galois group $G$. Assume that $G$ is a Frobenius group and $H$ is a Frobenius complement of $G$. Let $F(H)$ be the maximal normal nilpotent subgroup of $H$. If $H / F(H)$ is nilpotent, then every Artin L-function attached to an irreducible representation of $G$ arises from an automorphic representation over $F$, i.e., the Langlands' reciprocity conjecture is true for such Galois extensions.
\end{abstract}

\section{INTRODUCTION}

For any number field $F$, let $F_{\AA}$ be its adele ring. Let $E / F$ be a finite Galois extension of algebraic number fields, $G=\operatorname{Gal}(E / F)$, and $\operatorname{Irr}(G)$ be the set of isomorphism classes of irreducible complex representations of $G$. In the early 1900's, E. Artin associated an L-function $L(s, \rho, E / F)$, which is defined originally by an Euler product of the local L-functions over all nonarchimedean places of $F$, to every $\rho \in \operatorname{Irr}(G)$. These L-functions play a fundamental role in describing the distribution of prime ideal factorizations in such an extension, just as the classical L-functions of Dirichlet determine the distribution of primes in a given arithmetic progression and the prime ideal factorizations in cyclotomic fields. However, unlike the L-functions of Dirichlet, we do not know in general if the Artin L-functions extend to entire functions. In fact, we have the important:

Artin's conjecture. For any irreducible complex linear representation $\rho$ of $G$, $L(s, \rho, E / F)$ extends to an entire function whenever $\rho \neq 1$.

In the case $\rho=1$, it is to be observed that the corresponding L-function is the Dedekind zeta function $\zeta_{F}(s)$, which we know by the work of Hecke extends to a meromorphic function with only a simple pole at $s=1$.

In the simplest case when $\rho$ is one-dimensional, the Artin L-function equals a Hecke L-function attached to a grossencharacter given by class field theory. In fact, this equality of L-functions is called Artin's reciprocity law and represents one of the most marvelous discoveries of the human mind. It generalizes the celebrated law of quadratic reciprocity which corresponds to the case that $F=\mathbb{Q}$ and $E$ is a quadratic extension of $\mathbb{Q}$.

Received by the editors October 5, 1994

1991 Mathematics Subject Classification. Primary 11F39, 11R80, 11F70.

(C)1996 American Mathematical Society 
This singular fact, combined with an induction theorem for characters of a finite group $G$, enabled R. Brauer to show in the 1950's that every Artin L-function extends to a meromorphic function of $s$. (See [8], pp.121-128, for precise details.)

In some cases, group theory allows us to reduce Artin's conjecture to the onedimensional case, thereby allowing us to establish the conjecture in such fortuitous circumstances. For instance, if $G$ is supersolvable, then every irreducible representation is monomial (see [10], pp.66-67) and therefore by certain functorial properties of Artin L-functions (see [5], p. 233) we deduce Artin's conjecture.

In the late 1960's, R. Langlands had a remarkable insight about how to generalize the reciprocity law of Artin to the nonabelian context. After making precise the notion of an automorphic representation $\pi$ of $G L_{n}\left(F_{\mathbb{A}}\right)$, he associated to it an L-function, $L(s, \pi)$, which is also originally defined by an Euler product of local L-functions. Later, Godement and Jacquet showed that it extends to an entire function of $s$ if $\pi$ is cuspidal except for the case that $\pi$ is the trivial representation. In this paper we will use $L(s, \pi)$ to denote the nonarchimedean part of the Lfunction associated to $\pi$. We have the following important conjecture:

Langlands' reciprocity conjecture. For each irreducible representation $\rho$ of $\operatorname{Gal}(E / F)$ of dimension $n$, there exists a cuspidal automorphic representation $\pi=$ $\pi(\rho)$ of $G L_{n}\left(F_{\mathbb{A}}\right)$ such that

$$
L(s, \rho, E / F)=L(s, \pi) .
$$

Remarks. (1) Since $L(s, \pi)$ is an entire function, Langlands' reciprocity law implies Artin's conjecture. In the case $n=1$, an automorphic representation of $G L_{1}\left(F_{\mathbb{A}}\right)$ is just a grossencharacter and Langlands' reciprocity law recovers Artin's reciprocity law.

(2) To establish the equality of L-functions, it is enough to establish the equality of partial L-functions above which are over almost all finite places. The remark in [1], p.220, implies this. Also we can see this by applying Murty's "strong multiplicity one" result in [7] to the L-functions of both sides. Although the result in [7] is only for the Dirichlet series in the Selberg class where the Ramanujan condition on all places of $F$ is required, the proof of the "strong multiplicity one" result in [7] indeed only needs the Ramanujan condition on almost all places of $F$.

We will say that $\rho$ is automorphic if Langlands' reciprocity conjecture holds for $L(s, \rho, E / F)$. We will say that $G$ is of automorphic type if for every algebraic number field $F$ and every Galois extension $E$ of $F$ with $\operatorname{Gal}(E / F)=G$, we have that every irreducible representation $\rho$ of $G$ is automorphic over $F$. Thus the Artin reciprocity law implies that finite abelian groups are of automorphic type.

Langlands developed the important theory of base change for $G L_{2}$ to establish a fundamentally new reciprocity law. Combining his work with that of Tunnell [11] leads us to the following result: if $\operatorname{Gal}(E / F)$ is solvable and $\operatorname{dim} \rho \leq 2$, then $\rho$ is automorphic over $F$.

From these pinnacles of human thought, namely the Artin reciprocity law and the Langlands-Tunnell theorem, we extract:

Proposition 1. Let $E / F$ be a finite solvable extension of number fields and $\rho$ an irreducible complex linear representation of $\operatorname{Gal}(E / F)$. If $\operatorname{dim} \rho \leq 2$, then $\rho$ is automorphic over $F$.

We may parenthetically remark that it is precisely this result which is the starting point of Wiles' proof for the semi-stable case of the Taniyama-Shimura conjecture. 
These ideas have now been generalized in the work of Arthur and Clozel [1]. They proved that finite nilpotent groups are of automorphic type.

In this paper we will establish the reciprocity law for certain extensions whose Galois group is a Frobenius group. Recall that a group $G$ is called a Frobenius group if there is a subgroup $H \neq 1$ of $G$ such that $g^{-1} H g \cap H=1$ for all $g \in G \backslash H$. Frobenius groups are seldom nilpotent and so this result represents a significant enlargement of the class of groups of automorphic type.

Our main theorem is:

Theorem. Let $E / F$ be a finite Galois extension of algebraic number fields with its Galois group $G$ being a Frobenius group. Let $H$ be a Frobenius complement of $G$ and $F(H)$ be the maximal normal nilpotent subgroup of $H$. If $H / F(H)$ is nilpotent, then $G$ is of automorphic type.

\section{THE AUTOMORPHIC INDUCTION MAP}

The precise definitions of the base change map and the automorphic induction map were given by Arthur and Clozel in [1], p.119 and p.215. Since the proof of our theorem will mainly involve the automorphic induction map, we explain it when $\operatorname{Gal}(E / F)$ is cyclic with a prime order $l$.

Let $\Pi=\bigotimes \Pi_{w}$ and $\pi=\bigotimes \pi_{v}$ be automorphic representations of $G L_{n}$ over $E$ and $G L_{n l}$ over $F$ respectively. Let $w$ and $v$ be the places of $E$ and $F$ respectively, such that $w \mid v, v$ is unramified in $E$ and $\Pi_{w}, \pi_{v}$ are unramified. Let $t_{\Pi_{w}}$ and $t_{\pi_{v}}$ be the complex semisimple conjugacy classes associated by Langlands to $\Pi_{w}$ and $\pi_{v}$ respectively. Let $\zeta$ be a primitive $l^{\text {th }}$ root of unity.

Definition. $\pi$ is an automorphic induction of $\Pi$, if for all $w, v$ described above,

(i) when $v$ splits into $w_{1}, \cdots, w_{l}$, then

$$
t_{\pi_{v}}=t_{\Pi_{w_{1}}} \oplus \cdots \oplus t_{\pi_{w_{l}}},
$$

(ii) when $v$ is inert, then

$$
t_{\pi_{v}}=t_{\Pi_{w}}^{1 / l} \oplus \zeta t_{\Pi_{w}}^{1 / l} \oplus \cdots \oplus \zeta^{l-1} t_{\Pi_{w}}^{1 / l} .
$$

We say that an automorphic representation $\pi$ of $G L_{r}\left(F_{\mathbb{A}}\right)$ is induced from cuspidal, if there is an automorphic cuspidal unitary representation $\sigma$ of $M\left(F_{\mathbb{A}}\right)$, where $P=M N$ is an $F$-parabolic subgroup of $G L_{r}$, with its Levi factor $M$ and its unipotent radical $N$, such that

$$
\pi=\operatorname{Ind}_{M\left(F_{\mathrm{A}}\right) N\left(F_{\mathrm{A}}\right)}^{G\left(F_{\mathrm{A}}\right)} \sigma \otimes 1 .
$$

Proposition 2 (Arthur and Clozel [1, p.215]). Let $E / F$ be a Galois extension of prime degree $l$. Then, if $\Pi$ is a representation of $G L_{r}\left(E_{\mathbb{A}}\right)$ induced from cuspidal, there exists a unique automorphic representation $\pi$ of $G L_{r l}\left(F_{\mathbb{A}}\right)$ automorphically induced from $\Pi$. Moreover $\pi$ is induced from cuspidal.

Remark. From the definition, it is obvious that if $\pi$ is the automorphic induction of $\Pi$, then the local L-function of $\pi$ and that of $\Pi$ coincide at almost all places of $F$. Moreover from the proof of this proposition in [1], p.216, we can see what the $\pi$ is precisely. Actually, Arthur and Clozel defined the $\pi$ from their results of the base change. Using the proved relation of the L-function of automorphic representation 
and that of its base change image (see [1], p.60), we see that the local L-functions of $\Pi$ and $\pi$ coincide at every place of $F$ so that

$$
L(s, \Pi)=L(s, \pi) .
$$

Since Artin L-functions are invariant under the usual induction map, we can think of the automorphic induction map as the automorphic version of the induction map of Galois representations. Actually this is the starting point of studying the automorphic induction map.

Using Proposition 2, Arthur and Clozel proved the reciprocity law in the nilpotent case.

Proposition 3 ([1, p.220]). Let $E / F$ be a nilpotent extension of number fields. Then Langlands' reciprocity law is true.

\section{A Reciprocity LAW For some Frobenius extensions}

Proposition 4. Let $E / F$ be a solvable finite extension of number fields and $G=$ $\operatorname{Gal}(E / F)$. If there is a normal subgroup $N$ of $G$ satisfying

(1) $G / N$ is nilpotent,

(2) every irreducible representation of $N$ has dimension 1 or 2 , then $G$ is automorphic over $F$.

Proof. We use induction on the order of $G$. Since $G / N$ is nilpotent and $G$ is solvable, $G$ is a relative $M$-group with respect to $N$ (see [2], p.87). This means that for any $\rho \in \operatorname{Irr}(G)$, there is a subgroup $H$ of $G, N \leq H \leq G$, such that

$$
\rho=\operatorname{Ind}_{H}^{G} \phi, \quad \phi \in \operatorname{Irr}(H),\left.\quad \phi\right|_{N} \in \operatorname{Irr}(N) .
$$

In the above, $\operatorname{Ind}_{H}^{G} \phi$ is the induced representation from $\phi$, and $\left.\phi\right|_{N}$ is the restriction of $\phi$ to $N$.

If $H$ is not $G$, we can apply the induction on $H$, since $H$ satisfies conditions (1) and (2) in the proposition. Hence

$$
L(s, \rho)=L\left(s, \phi, E / E^{H}\right)=L(s, \Pi),
$$

where $E^{H}$ is the subfield of $E$ fixed by $H$, and $\Pi$ is an automorphic cuspidal representation of $G L_{n}\left(\left(E^{H}\right)_{\mathbb{A}}\right)$. Since $G / N$ is nilpotent, there is a chain of subgroups of $G$

$$
N_{0}=N \triangleleft N_{1} \triangleleft N_{2} \triangleleft \cdots \triangleleft N_{k-1} \triangleleft N_{k}=G,
$$

such that $N_{i-1}$ is a normal subgroup of $N_{i}$ and $N_{i} / N_{i-1}$ has a prime order, for $1 \leq i \leq k$. By applying finitely many steps of the automorphic induction on the chain of the subfields corresponding to the chain of subgroups above, we know that there is an automorphic representation $\pi$ of $G L_{r}\left(F_{\mathbb{A}}\right)$ such that

$$
L(s, \Pi)=L(s, \pi),
$$

by Proposition 2 and the remark following it. Since $\rho$ is irreducible, the $\pi$ must be cuspidal by Theorem 4.7 in [4]. Thus the proof is done if $H$ is not $G$.

If $H=G$, then $\left.\rho\right|_{N} \in \operatorname{Irr}(N)$. Since every irreducible representation of $N$ has dimension one or two, $\rho$ has dimension one or two. Therefore by Proposition 1, $\rho$ is automorphic over $F$.

Let us record some basic properties of Frobenius groups we will use in the following proposition. 
Proposition 5. Let $G$ be a finite Frobenius group.

(1) $G=K H$, where $K \triangleleft G, K$ is nilpotent, both $K$ and $H$ are Hall subgroups of $G$ and $(|K|,|H|)=1$.

(2) Every Sylow subgroup of $G$ is a cyclic or generalized quaternion group.

(3) Let $\rho \in \operatorname{Irr}(G)$. If the kernel $\operatorname{Ker}(\rho)$ of $\rho$ does not contain $K$, then $\rho=$ $\operatorname{Ind}_{K}^{G} \sigma, \sigma \in \operatorname{Irr}(K)$.

Since, for a Frobenius group $G$, the subgroup $K$ of $G$ stated in the proposition above is uniquely determined by $G$, we call $K$ the Frobenius kernel of $G$. The subgroup $H$ of $G$ in the proposition is unique up to conjugation in $G$; we call $H$ a Frobenius complement of $G$. For the proof of this, see [9], pp.179-228.

Theorem. Let $E / F$ be a finite Galois extension of algebraic number fields with its Galois group $G$ being a Frobenius group. Let $H$ be a Frobenius complement of $G$ and $F(H)$ be the maximal normal nilpotent subgroup of $H$. If $H / F(H)$ is nilpotent, then $G$ is of automorphic type.

Proof. Let $\rho \in \operatorname{Irr}(G)$, and $K$ be the Frobenius kernel of $G$.

If $\operatorname{ker}(\rho)$ does not contain $K$, then by (3) in Proposition 5, $\rho=\operatorname{Ind}_{K}^{G} \sigma, \sigma \in$ $\operatorname{Irr}(K)$. So

$$
L(s, \rho, E / F)=L\left(s, \sigma, E / E^{K}\right),
$$

where $E^{K}$ is the subfield of $E$ fixed by $K$. Since $K$ is nilpotent, $\rho$ is automorphic over $E^{K}$, by Proposition 3 . Since $K$ is normal in $G, E^{K} / F$ is a Galois extension and $\operatorname{Gal}\left(E^{K} / F\right)=G / K=H$. Because both $F(H)$ and $H / F(H)$ are solvable, $H$ is solvable. So we can apply the automorphic induction finitely many times to obtain that $\rho$ is automorphic over $F$.

If $\operatorname{ker}(\rho)$ contains $K$, we can regard $\rho \in \operatorname{Irr}(H)$. The result in our theorem follows from the following lemma which deals with a more general situation than our case.

Lemma. Let $G=\operatorname{Gal}(E / F)$ be a finite solvable group. Suppose that every Sylow $p$-subgroup of $G$ is abelian for $p>2$ and every irreducible representation of Sylow 2subgroups has dimension one or two. If $G / F(G)$ is nilpotent, then $G$ is automorphic over $F$.

Proof of the Lemma. We claim that every irreducible representation of $F(G)$ is of dimension one or two. In fact, since $F(G)$ is nilpotent, $F(G)$ is the direct product of its Sylow subgroups, hence for $\rho \in \operatorname{Irr}(F(G)), \rho$ is a tensor product of irreducible representations of the Sylow subgroups of $F(G)$. Since every irreducible representation of the Sylow $p$-subgroups has dimension one for $p>2$, and dimension one or two for $p=2$, every irreducible representation of $F(G)$ has dimension one or two. In this way, we have proved the claim. By this claim and Proposition 4, we have then proved the lemma.

Now for our $H$, its Sylow subgroups are cyclic or generalized quaternion groups, and hence the Sylow $p$-subgroup of $F(H)$ is cyclic for $p>2$. Because every subgroup of a generalized quaternion group is also a cyclic or generalized quaternion group, the Sylow 2-subgroup of $F(H)$ is cyclic or generalized quaternion. Since a generalized quaternion group $Q$ has a cyclic or abelian normal subgroup of index 2 , so every irreducible representation of $Q$ has dimension one or two. To see this, see [2], p.203, or compute directly. So, by the lemma we have finished the proof of the theorem. 
Remark. In the theorem above if we assume that $H$ is supersolvable, then the commutator subgroup $H^{\prime}$ of $H$ is nilpotent, hence $H^{\prime} \leq F(H)$. Therefore $H / F(H)$ is abelian and hence is nilpotent. So this situation is already included in the theorem above. Note that if the order of $H$ is odd, then $H$ is supersolvable, thus $G$ is automorphic over $F$ in this case. In particular, if $G$ is a Frobenius group with odd order, then $G$ is automorphic over $F$.

\section{ACKNOWLEDGMENT}

I wish to thank Professor Ram Murty for directing my attention to Langlands' reciprocity law and for his invaluable encouragement and support. I also wish to thank the referee for suggestions, especially for those on the introduction of this paper and on an application of Murty's "strong multiplicity one" result of [7].

\section{REFERENCES}

1. J. Arthur and L. Clozel, Simple Algebras, Base Change, and the Advanced Theory of the Trace Formula, Annals of Mathematics Studies, Princeton University Press, 1989. MR 90m:22041

2. M. Isaacs, Character Theory of Finite Groups, Academic Press, 1976. MR 57:417

3. H. Jacquet, and J. Shalika, On Euler Products and the Classification of Automorphic Representations I, Amer. J. Math. 103, No. 3 (1981), 499-558. MR 82m:10050a

4. H. Jacquet, and J. Shalika, On Euler Products and the Classification of Automorphic Representations II, Amer. J. Math. 103, No. 3 (1981), 777-815. MR 82m:10050b

5. S. Lang, Algebraic Number Theory, Second Edition, Graduate Texts in Math., no. 110, Springer-Verlag, New York, 1994. MR 95f:11085

6. R. Langlands, Base Change for GL(2), Annals of Mathematics Studies, Princeton University Press, 1980. MR 82a:10032

7. R. Murty and K. Murty, Strong Multiplicity One for Selberg's Class, Commptes Rendus Acad. Sci. Paris, Vol. 319, Series I (1994), 315-320. MR 95h:11094

8. J. Neukirch, Class Field Theory, Springer, 1986. MR 87i:11005

9. D. Passman, Permutation Groups, Benjamin,inc. 1968. MR 38:5908

10. J.-P. Serre, Linear representations of finite groups, springer, 1977. MR 56:8675

11. J. Tunnell, Artin's conjecture for representations of octahedral type, Bulletin (New Series) of the American Mathematical Society, Vol.5, No.2, 1981. MR 82j:12015

Mathematical Sciences Research Institute, 1000 Centennial Drive, Berkeley, CaliFORNIA 94720

E-mail address: yuanli@msri.org

Current address: Department of Mathematics, Purdue University, West Lafayette, Indiana 47907

E-mail address: yz@math.purdue.edu 